\title{
Confronting GW190814 with hyperonization in dense matter and hypernuclear compact stars
}

\author{
Armen Sedrakian, ${ }^{1,2, *}$ Fridolin Weber, ${ }^{3,4}$ and Jia $\mathrm{Jie} \mathrm{Li}^{5}$ \\ ${ }^{1}$ Frankfurt Institute for Advanced Studies, D-60438 Frankfurt-Main, Germany \\ ${ }^{2}$ Institute of Theoretical Physics, University of Wroctaw, 50-204 Wroctaw, Poland * \\ ${ }^{3}$ Department of Physics, San Diego State University, San Diego, California 92182, USA \\ ${ }^{4}$ Center for Astrophysics and Space Sciences, University of California, San Diego, La Jolla, California 92093, USA ${ }^{\dagger}$ \\ ${ }_{5}^{5}$ Institute for Theoretical Physics, J. W. Goethe University, D-60438 Frankfurt am Main, Germany
}

(Dated: August 12, 2020)

\begin{abstract}
We examine the possibility that the light companion in the highly asymmetric binary compact object coalescence event GW190814 is a hypernuclear star. We use density functional theory with functionals that have been tuned to the properties of $\Lambda$ hypernuclei as well as astrophysical constraints placed by the masses of the most massive millisecond pulsars, the mass-radius range inferred from the NICER experiment, and the binary neutron star merger event GW170817. We compute general-relativistic static and maximally rotating Keplerian configurations of purely nucleonic and hypernuclear stars. We find that while nucleonic stars are broadly consistent with a neutron star being involved in GW190814, this would imply no new degrees of freedom in the dense matter up to 6.5 times the nuclear saturation density. Allowing for hyperonization of dense matter, we find that the maximal masses of hypernuclear stars, even for maximal rapidly rotating configurations, are inconsistent with a stellar nature interpretation of the light companion in GW190814, implying that this event involved two black holes rather than a neutron star and a black hole.
\end{abstract}

\section{INTRODUCTION}

The recent measurement by the LIGO-Virgo Collaboration (hereafter LVC) [1] of gravitational waves from a binary coalescence of a $24.3 M_{\odot}$ black hole with a compact object in the mass the range of $2.50-2.67 M_{\odot}$ has raised interest in the question of whether the light companion is the heaviest known neutron star (NS) or the lightest known black hole $(\mathrm{BH})$. The mass of this object falls into the so-called "mass gap" $2.5 \leq M / M_{\odot} \leq 5$ where neither a neutron star nor a black hole have been observed so far. This observation poses yet another challenge to the theoretical models of dense nuclear matter in the light of gravitational wave observations; for reviews see $[2,3]$.

Theoretically, the modern density functional models, which are compatible with the nuclear phenomenology in the vicinity of nuclear saturation density and satisfy the mass and/or radius constraints coming from measurements of massive $\left(\sim 2.0 M_{\odot}\right)$ pulsars [4], the NICER experiment [5, 6], and the multimessenger GW170817 event [7-9], predict maximum masses of static neutron star sequences of the order of $2.50 M_{\odot}$. As is well known, the maximum mass of a rapidly rotating $\mathrm{NS}$ is around $20 \%$ larger than its static counterpart [10-13]. Therefore the tension between the NS interpretation of the light companion in GW190814 and the maximal masses predicted by nuclear models is mitigated if the light companion were a rapidly rotating NS. At the same time, the $\mathrm{BH}$ origin of a compact object within the mass gap

\footnotetext{
*Electronic address: sedrakian@fias.uni-frankfurt.de

†Electronic address: fweber@sdsu.edu, fweber@ucsd.edu
}

range is possible through the prior coalescence of a binary NS or an NS-white dwarf system, which would suggest that GW190814 originates from a triple star system. Ideas have already been put forward regarding the nature of the light companion of GW190814 which invoke (stationary or rapidly rotating) very massive NS composed of nucleonic matter [14-18] at the instance of coalescence or in the past.

In this paper we examine the possibility that the lighter companion in GW190814 is a hypernuclear star and show that hyperonization in dense matter is incompatible with its interpretation as a NS, even if one considers a maximally fast rotating (Keplerian) hypernuclear star. Thus we conclude that the light companion in GW190814 is a black hole if hyperonization takes place in compact stars.

The hyperonization of dense matter is based on a robust energetic argument which asserts that hyperons will nucleate in dense nuclear matter once the neutron Fermi energy reaches the (in-medium) rest masses of hyperons. Although hyperonization in dense matter has been studied for several decades (for early studies see, for example, [19-21]), only during recent years covariant density functional models were developed which were compatible with the hypernuclear data, mass and/or radius measurements of neutron stars and the tidal deformability inferred from the GW170817 event [22-30]. In this work we use a covariant density functional model whose parameters are adjusted to available hypernuclear and astrophysical data and show that both the maximal masses of hypernuclear stars in both the static and Keplerian limits are incompatible with the mass range inferred for the light companion in the GW190814 event. There are a few alternatives to the hyperonization scenario discussed here: (a) deconfined quark matter phases may appear before the hyperonization threshold; (b) the $\Delta$ resonances 
may appear in addition to hyperons. The first effect may have profound implications for the equation of state and structure of NS and clearly requires a separate discussion (see [30-35] for recent discussions of various phases and resulting features of compact objects). The appearance of $\Delta$ resonances does not affect the maximum mass of a static NS, but can reduce the radius of the star by tens of percent ([28] and references therein). Because our arguments are based on the static and Keplerian maximum masses of hypernuclear stars which are very close to those derived for $\Delta$-admixed hypernuclear matter, our conclusions will not be affected in this case.

\section{EQUATION OF STATE OF HYPERNUCLEAR STARS}

For our study we used two equations of state of hypernuclear matter obtained from covariant density functional theory: the first is based on a functional with the density-dependent meson-baryon couplings with DDME2 parametrization and its extension to the hypernuclear sector $[25,28,36,37]$. As an alternative equation of state, we used the NL3 [38] model and its extension to the hyperonic sector.

The Lagrangian density of matter can be written as $\mathcal{L}=\mathcal{L}_{B}+\mathcal{L}_{l}$, where the baryonic contribution is given by

$$
\begin{aligned}
\mathcal{L}_{B} & =\sum_{B} \bar{\psi}_{B}\left[\gamma^{\mu}\left(i \partial_{\mu}-g_{\omega N} \omega_{\mu}-\frac{1}{2} g_{\rho N} \boldsymbol{\tau} \cdot \boldsymbol{\rho}_{\mu}\right)\right. \\
& \left.-\left(m_{B}-g_{\sigma N} \sigma\right)\right] \psi_{B}+\frac{1}{2} \partial^{\mu} \sigma \partial_{\mu} \sigma-\frac{1}{2} m_{\sigma}^{2} \sigma^{2} \\
& -\frac{1}{4} \omega^{\mu \nu} \omega_{\mu \nu}+\frac{1}{2} m_{\omega}^{2} \omega^{\mu} \omega_{\mu}-\frac{1}{4} \boldsymbol{\rho}^{\mu \nu} \boldsymbol{\rho}_{\mu \nu}+\frac{1}{2} m_{\rho}^{2} \boldsymbol{\rho}^{\mu} \cdot \boldsymbol{\rho}_{\mu},
\end{aligned}
$$

where $B$ sums over baryons and $\psi_{B}$ are the baryonic Dirac fields with masses $m_{B}$. The meson fields $\sigma, \omega_{\mu}$, and $\boldsymbol{\rho}_{\mu}$ mediate the interactions among the baryon fields, $\omega_{\mu \nu}$ and $\boldsymbol{\rho}_{\mu \nu}$ represent the field strength tensors of vector mesons, and $m_{\sigma}, m_{\omega}$, and $m_{\rho}$ are their masses. The baryon-meson coupling constants are denoted by $g_{i B}$ with $i=\sigma, \omega, \rho$. The leptonic contribution is given by $\mathcal{L}_{l}=\sum_{\lambda} \bar{\psi}_{\lambda}\left(i \gamma^{\mu} \partial_{\mu}-m_{\lambda}\right) \psi_{\lambda}$, where $\lambda$ sums over the leptons $e^{-}$and $\mu^{-}$, which are treated as free Dirac fields with masses $m_{\lambda}$. In the DDME2 model the coupling constants in the nucleonic Lagrangian are density dependent and are parametrized according to the relation $g_{i N}\left(n_{B}\right)=g_{i N}\left(n_{s}\right) h_{i}(x)$, for $i=\sigma, \omega$, and $g_{\rho N}\left(n_{B}\right)=$ $g_{\rho N}\left(n_{0}\right) \exp \left[-a_{\rho}(x-1)\right]$ for the $\boldsymbol{\rho}_{\mu}$-meson, where $n_{B}$ is the baryon density, $n_{0}$ is the nuclear saturation density, and $x=n_{B} / n_{0}$. This parametrization has in total eight parameters, which are adjusted to reproduce the properties of symmetric and asymmetric nuclear matter and the binding energies, charge radii, and neutron radii of spherical nuclei (see [28]). Its predictions for the main characteristic parameters for nuclear matter at saturation density are $K=251.15 \mathrm{MeV}$ for compressibility,
$E_{\mathrm{sym}}=32.31 \mathrm{MeV}$ for symmetry energy, $L_{\mathrm{sym}}=51.27$ $\mathrm{MeV}$ for the slope of the symmetry energy.

In the hypernuclear sector, the vector meson-hyperon couplings are given by the $S U(6)$ flavor symmetric quark model, whereas the scalar meson-hyperon couplings are determined by fits to empirical hypernuclear potentials: $U_{\Lambda}=U_{\Sigma}=-30 \mathrm{MeV}, U_{\Xi}=14 \mathrm{MeV}$ at nuclear saturation density. Note that the Lagrangian of this model has only linear meson-field interaction terms and the nucleonmeson coupling constants are density dependent. The NL3 model is used as an alternative model, which has density independent meson-nucleon couplings, but contains nonlinear in meson fields terms; we will comment on the differences between these models. For illustration, we will also consider hypernuclear stars which contain a $\Delta$-resonance component, again within the DDME2 model [28].

The equations of state of purely nucleonic matter and hypernuclear matter are illustrated in Fig. 1. The

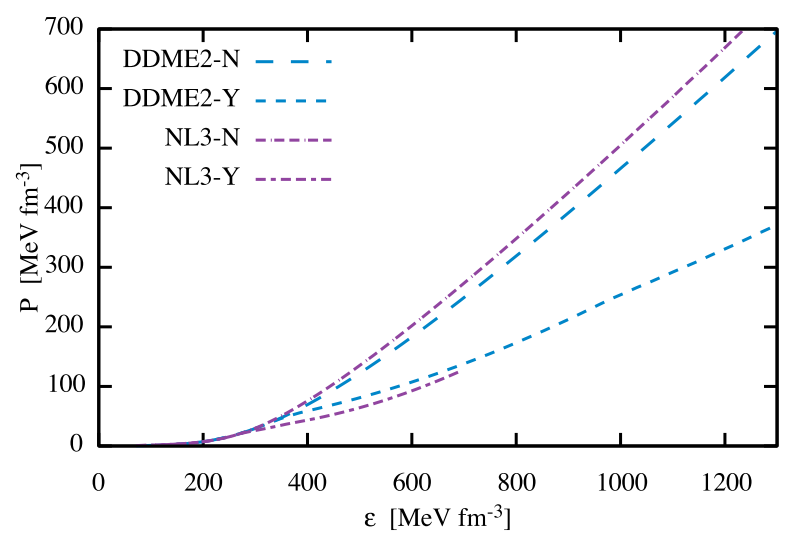

FIG. 1: The equation of state of dense matter for two cases: purely nucleonic matter $(\mathrm{N})$ and hypernuclear matter $(\mathrm{Y})$ which includes the full baryon octet. The long- and shortdashed lines show the results for the DDME2 parametrization. The dash-dotted and short-long-dashed lines show the same for the NL3 parametrization. Note the softening of the equation of state (reduction of the pressure) in each case caused by the onset of hyperonization.

composition of hypernuclear matter computed for the DDME2 parametrization is shown in Fig. 2. It is seen that, in the relevant density range, the $\Sigma$-hyperons do not appear and that the dominant hyperonic component is the $\Lambda$-hyperon. Among the hyperons, the $\Lambda$ hyperon properties are best constrained due to the tuning of the interactions (in particular the coupling to the $\sigma$ meson) to the $\Lambda$ single and double hypernuclei [25, 37]. The variations in the magnitude of the $\Lambda$-hyperon potential in nuclear matter within various extensions of the DDME2 model to the hypernuclear sector are too small to affect the stellar properties and the equation of state $[25,27,28,37]$. 


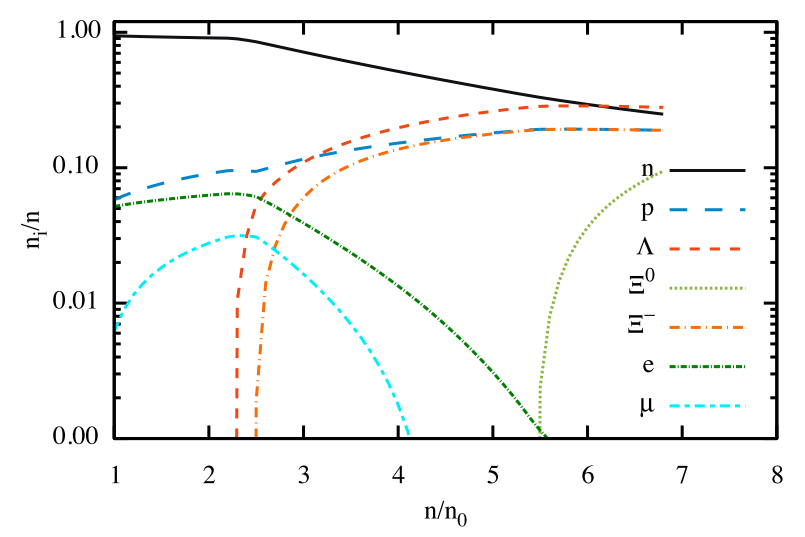

FIG. 2: Particle fractions $n_{i} / n$ ( $i$ refers to baryons in the baryon octet) in hypernuclear matter according to the DDME2 model. Here $n$ denotes baryon density, and $n_{0}$ is the saturation density of ordinary nuclear matter. The results for NL3 model show the same features and are not shown here.

\section{MASS-RADIUS RELATIONS OF COMPACT STARS}

The general relativistic structure equations of compact stars [39, 40] for the hypernuclear model equations of state shown in Fig. 1 were solved for spherically symmetric (nonmagnetized) stars in the cases of static (nonrotating) and maximally rotating (Keplerian) stars. The rotating configurations were generated using the public domain RNS code ${ }^{1}$. Note that in both cases the stable configurations of hypernuclear stars are determined by the Bardeen-Thorne-Meltzer criterion [41], which implies that a star is stable only as long as its mass is increasing with the central density. Thus, along the rising branch on the mass-radius curve the stars with the maximum mass for any given sequences are the last stable configurations.

In Fig. 3 we show the mass-radius relations of nucleonic and hypernuclear stars based on the DDME2 parametrization for static and maximally rotating configurations. Nucleonic models in the static case reach a maximum mass of $2.48 M_{\odot}$ (with a radius of $R=12.1$ $\mathrm{km}$ ), which makes them marginally compatible with the NS interpretation of the light companion of GW190814. The maximally rotating nucleonic models reach masses up to $3 M_{\odot}$ and thus comfortably account for a NS in GW190814. The sequences for the NL3 model show the same behavior whereby the maximum mass of nucleonic stars is larger whereas that of hypernuclear stars is lower than in the DDME2 model. This implies that our arguments are even stronger enforced by the NL3 model. For comparison, we also show the sequences of static and

\footnotetext{
1 www.gravity.phys.uwm.edu/rns/
}

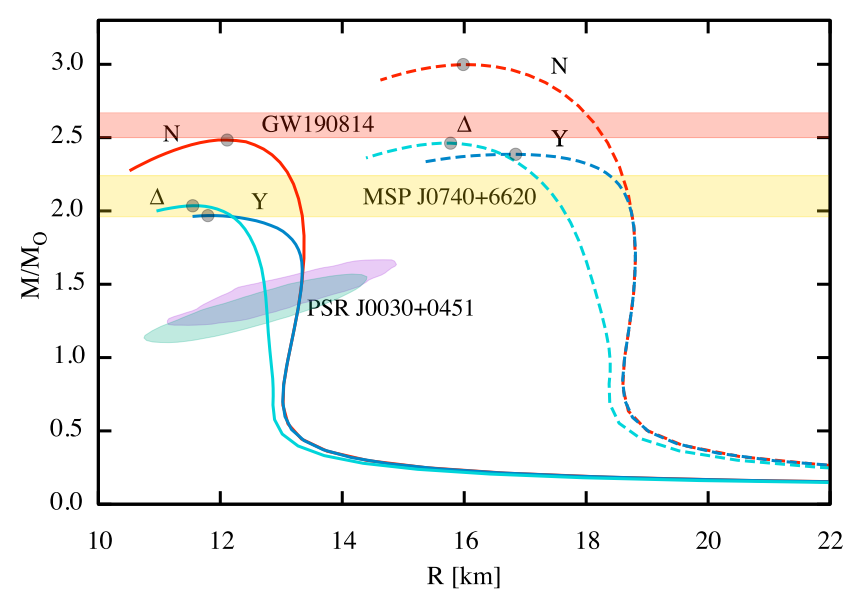

FIG. 3: The mass-radius relations for nonrotating (solid lines) and maximally rotating (dashed lines) nucleonic $(N)$, hypernuclear $(Y)$ and $\Delta$-admixed-hypernuclear $(\Delta)$ stars. The colored areas show the constraints inferred from the most massive pulsar MSP J0740+6620 [4], the mass-radius limits inferred from the NICER experiment $[5,6]$ and the mass limits from GW190814 [1]. The circles indicate the maximum masses of the sequences, to the left of which the stars are unstable.

maximally rotating $\Delta$-resonance admixed-hypernuclear star with the same DDME2 model [28]; as anticipated the maximum masses in this case are not modified substantially, but the radii of the models are smaller than for nucleonic and hypernuclear models.

The nucleonic models satisfy the complementary to GW190814 constraints, specifically, the lower bound on the maximum mass placed by MSP J0740+6620 [4], and the mass-radius limits inferred from the NICER experiment $[5,6]$ giving, for example, a radius of $R=13.3$ $\mathrm{km}$ for a $M=1.33 M_{\odot}$ star. Turning to the hypernuclear models, we note that the softening of the equation of state triggered by the hyperonization leads to a lower maximum mass compared to the nucleonic case. The maximum mass of static hypernuclear stars is $M \simeq 2.0 M_{\odot}$ as is thus consistent with the massive pulsar MSP J0740+6620, but clearly is inconsistent with a NS in GW190814. The maximally rotating Keplerian models of hypernuclear stars have maximum masses $\leq 2.3 M_{\odot}$. This implies that the maximal rotation is not sufficient to raise masses of hypernuclear stars to the required value $2.5 M_{\odot}$. Thus, we conclude that independent of the rotation rate the hypernuclear stars modeled with the DDME2 and NL3 density functionals are incompatible with the light companion of GW190814 being an NS. While one can attempt to resurrect the compatibility by modifying the coupling constant for example in the vector-meson sector by going from $S U(6)$ to $S U(3)$ symmetry (see, e.g., [42]), we anticipate that the tension between the theory and the data will still remain.

Finally, in Fig. 4 we show the tidal deformability vs mass relations of nucleonic and hypernuclear stars 


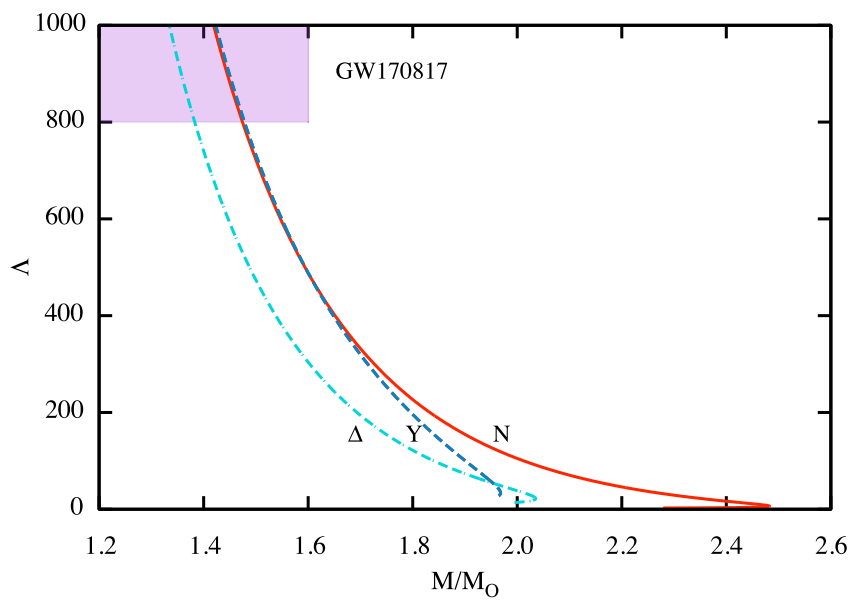

FIG. 4: The tidal deformability of static nucleonic (solid line), hypernuclear (dashed), and $\Delta$-admixed-hypernuclear stars (dash dotted) according to the DDME2 model. The colored area shows the $90 \%$ confidence exclusion region for tidal deformability of equal mass binary obtained from the analysis of GW170817 for the range of NS masses $1.17 \leq M / M_{\odot} \leq 1.6$.

based on the DDME2 parametrization for static configurations along with the $90 \%$ confidence exclusion region for equal mass NS-NS binary obtained from the analysis of GW170817. Our main point here is seen that the massive stars have very small tidal deformability which is not expected to be observable at the current level of the sensitivity of gravitational wave observatories. Note also that the mild discrepancy between the prediction of the tidal deformability of the DDME2 model for lighter (canonical) mass NS and the inference from GW170817 can be cured by accounting for the onset of $\Delta$ resonances [28], as illustrated in Fig. 4, or adjusting higher-order expansion parameters of the density functional [29].

\section{CONCLUSIONS AND OUTLOOK}

In this work, we investigated the possibility that the light companion in the GW190814 event is a hypernuclear compact star. The equation of state was taken from studies of covariant density functional theory of hypernuclear matter with the parameters tuned to $\Lambda$ hypernuclear data and astrophysical constraints imposed by the massive pulsars, NICER experiment, and GW170817 event. We have considered both static configurations and maximally fast rotating configurations of hypernuclear stars. As expected, the purely nucleonic stars are consistent with the involvement of NS in GW190814 even if the star was nonrotating; adding some degree of rotation would make the nucleonic models broadly compatible with the scenario involving an NS. However, such interpretation would imply that matter maintains its purely nucleonic degrees of freedom up to densities 6.5 times the nuclear saturation. A more likely and robust scenario is the hyperonization of dense matter, in which heavier members of the baryon octet nucleate once their (in-medium) masses become of the order of the neutron chemical potential. We found that if hyperonization takes place, then the maximal masses of sequences of hypernuclear stars are well below the lower bound inferred for the light companion in GW190814 for particular density functional studied. The discrepancy can be mitigated by modifying the coupling constant for example in the vector-meson sector by going from $S U(6)$ to $S U(3)$ or varying the $\Xi^{-}$potential, but we anticipate that the tension between the observation and the theory will remain - unless extreme assumptions are not made about couplings entering the density functionals. The characteristic parameters of symmetric nuclear matter (compressibility, symmetry energy, and the slope of the symmetry energy) at saturation density are well reproduced by the DDME2 model (this is not the case for NL3 model which has a large slope of the symmetry energy), therefore the nucleonic sector can be modified only by adjusting the not-well-constrained parameters corresponding to higher-order terms in the expansions around the saturation density.

Thus, our main conclusion is that the hyperonization of dense matter acts strongly against the interpretation of GW190814 involving a neutron star. It should be noted that the hypernuclear models are otherwise broadly consistent with the masses inferred for most massive pulsars and the mass-radius range for canonical mass neutron stars inferred by the NICER experiment, see Fig. 3. Furthermore, the models we used have been shown to be compatible with the constraints on tidal deformability of a compact star as inferred from the GW170817 event, see in particular [29].

We have not addressed in detail the possibility of either phase transition to quark matter or $\Delta$-resonance admixture in the hypernuclear matter. The first issue needs substantial changes in the input physics, therefore, we do not comment here, rather than refer to recent studies in Refs. [30-35]. Adding $\Delta$-resonances to hypernuclear matter does change the composition of matter and the radius of the star, but does not increase (maximum) masses of stellar sequences significantly (see Fig. 3).

\section{Acknowledgments}

A.S. is supported by the Deutsche Forschungsgemeinschaft (Grant No. SE 1836/5-1) and European COST Actions "PHAROS" (CA16214). F.W. is supported through the U.S. National Science Foundation under Grant PHY-171406. We are grateful to M. Alford, D. Blaschke, A. Harutyunyan, M. Oertel, A. Raduta, and M. Sinha for discussions. 
[1] R. Abbott et al. (LIGO Scientific, Virgo Collaborations), Astrophys. J. 896, L44 (2020), arXiv:2006.12611

[2] L. Baiotti, Prog. Part. Nucl. Phys. 109, 103714 (2019), arXiv:1907.08534

[3] K. Chatziioannou, (2020), arXiv:2006.03168

[4] H. T. Cromartie et al., Nat. Astron. 4, 72 (2019), arXiv:1904.06759

[5] M. C. Miller et al., Astrophys. J. Lett. 887, L24 (2019), arXiv:1912.05705

[6] T. E. Riley et al., Astrophys. J. Lett. 887, L21 (2019), arXiv:1912.05702

[7] B. P. Abbott, R. Abbott, R. X. Adhikari, A. Ananyeva, S. B. Anderson, et al., Astrophys. J. Lett. 848, L12 (2017)

[8] B. P. Abbott, R. Abbott, T. D. Abbott, F. Acernese, K. Ackley, et al., Astrophys. J. Lett. 848, L13 (2017)

[9] B. P. Abbott, R. Abbott, T. D. Abbott, F. Acernese, K. Ackley, et al. (LIGO Scientific Collaboration and Virgo Collaboration), Phys. Rev. Lett. 119, 161101 (2017)

[10] F. Weber and N. K. Glendenning, Astrophys. J. 390, 541 (1992)

[11] G. Cook, S. Shapiro, and S. Teukolsky, Astrophys. J. 424, 823 (1994)

[12] S. Bonazzola, E. Gourgoulhon, and J.-A. Marck, Phys. Rev. D 58, 104020 (1998), arXiv:astro-ph/9803086

[13] V. Paschalidis and N. Stergioulas, Living Rev. Relativity 20, 7 (2017), arXiv:1612.03050

[14] E. R. Most, L. J. Papenfort, L. R. Weih, and L. Rezzolla, (2020), arXiv:2006.14601

[15] F. J. Fattoyev, C. J. Horowitz, J. Piekarewicz, and B. Reed, (2020), arXiv:2007.03799

[16] A. Tsokaros, M. Ruiz, and S. L. Shapiro, (2020), arXiv:2007.05526

[17] N.-B. Zhang and B.-A. Li, (2020), arXiv:2007.02513

[18] I. Tews, P. T. H. Pang, T. Dietrich, M. W. Coughlin, S. Antier, M. Bulla, J. Heinzel, and L. Issa, (2020), arXiv:2007.06057

[19] V. A. Ambartsumyan and G. S. Saakyan, Sov. Astron. 4, 187 (1960)

[20] N. K. Glendenning, Astrophys. J. 293, 470 (1985)

[21] F. Weber, Pulsars as Astrophysical Laboratories for $\mathrm{Nu}$ clear and Particle Physics (Institute of Physics, Bristol, U.K., 1999)

[22] M. Oertel, C. Providência, F. Gulminelli, and A. R.
Raduta, J. Phys. G 42, 075202 (2015), arXiv:1412.4545

[23] M. Fortin, C. Providência, A. R. Raduta, F. Gulminelli, J. L. Zdunik, P. Haensel, and M. Bejger, Phys. Rev. C 94, 035804 (2016), arXiv:1604.01944 [astro-ph.SR]

[24] C. Providência, M. Fortin, H. Pais, and A. Rabhi, Front. in Astron. Space Sci. 6, 13 (2019), arXiv:1811.00786

[25] M. Fortin, A. R. Raduta, S. Avancini, and C. Providência, Phys. Rev. D 101, 034017 (2020), arXiv:2001.08036

[26] L. Tolos, M. Centelles, and A. Ramos, Astrophys. J. 834, 3 (2017), arXiv:1610.00919

[27] A. R. Raduta, A. Sedrakian, and F. Weber, Mon. Not. R. Astron. Soc. 475, 4347 (2018), arXiv:1712.00584

[28] J. J. Li, A. Sedrakian, and F. Weber, Phys. Lett. B $\mathbf{7 8 3}$ $234(2018)$

[29] J. J. Li and A. Sedrakian, Astrophys. J. Lett. 874, L22 (2019), arXiv:1904.02006

[30] J. J. Li, A. Sedrakian, and M. Alford, Phys. Rev. D 101, $063022(2020)$

[31] J.-E. Christian and J. Schaffner-Bielich, Astrophys. J. Lett. 894, L8 (2020), arXiv:1912.09809

[32] J. P. Pereira, M. Bejger, N. Andersson, and F. Gittins, Astrophys. J. 895, 28 (2020), arXiv:2003.10781

[33] M. Ferreira, R. C. Pereira, and C. Providência, Phys. Rev. D 101, 123030 (2020), arXiv:2005.10543

[34] D. Blaschke, A. Ayriyan, D. E. Alvarez-Castillo, and H. Grigorian, Universe 6, 81 (2020), arXiv:2005.02759

[35] A. Bauswein, S. Blacker, V. Vijayan, N. Stergioulas, K. Chatziioannou, J. A. Clark, N.-U. F. Bastian, D. B. Blaschke, M. Cierniak, and T. Fischer, (2020), arXiv:2004.00846

[36] G. Colucci and A. Sedrakian, Phys. Rev. C 87, 055806 (2013)

[37] E. van Dalen, G. Colucci, and A. Sedrakian, Phys. Lett. B 734, 383 (2014)

[38] G. A. Lalazissis, J. König, and P. Ring, Phys. Rev. C 55, 540 (1997), arXiv:nucl-th/9607039

[39] R. C. Tolman, Phys. Rev. 55, 364 (1939)

[40] J. R. Oppenheimer and G. M. Volkoff, Phys. Rev. 55, 374 (1939)

[41] J. M. Bardeen, K. S. Thorne, and D. W. Meltzer, Astrophys. J. 145, 505 (1966)

[42] J. J. Li, W. H. Long, and A. Sedrakian, Eur. Phys. J. A 54, $133(2018)$ 